review

\title{
Radiation effects on skeletal muscle
}

\author{
Mihaela Jurdana ${ }^{1,2}$ \\ ${ }^{1}$ College of Health Care Izola, University of Primorska; \\ ${ }^{2}$ Science and Research Centre Koper, University of Primorska, Slovenia
}

\begin{abstract}
Background. Adult skeletal muscle is considered resistant to ionizing radiation unless higher doses of radiation are applied; a fact that is attributed to the low number of radiosensitive proliferating cells in adulthood. However, developing skeletal muscles are highly sensitive to ionizing radiation, thus radiotherapy in childhood may induce muscular atrophy. Radiation affects muscle satellite cells by impairing their activation, proliferation and differentiation, as well as neuromuscular junction, by influencing the ionic membrane permeability affecting the $\mathrm{Na}^{+} / \mathrm{K}^{+}$pump. It also prevents muscle growth during development and after injury.

Conclusions. The results of the investigation performed after radiation point to the occurrence of a significant change in muscle satellite cell activity. Inhibitors of some proteins such as cytokines in muscle satellite cells could provide a therapeutic benefit in diseases for which muscle mass is limiting, improve response to cancer therapy, and increase life span in patients with cachexia.
\end{abstract}

Key words: radiation effects; neuromuscular junction; muscle satellite cells; aging.

\section{Introduction}

The cells of normal tissue are not independent but form a complete integrated structure. Cell death after irradiation occurs mostly as cells attempt to divide. In tissues with a rapid turnover rate, damage quickly becomes evident - in a matter of hours and days after radiation. In tissues in which cells divide rarely, radiation damage to cells may remain latent for a long time and can be expressed very slowly. Radiation damage to cells that are already on the path to differentiation and are not in any case planning to divide many times is of little consequence. Radiation

Received 15 February 2008

Accepted 5 March 2008

Correspondence to: Mihaela Jurdana, PhD; University of Primorska, College of Health Care Izola, Polje 42, 6310 Izola, Slovenia. Phone: +386 566264 69; Fax: +3865662 64 80; E-mail: mihaela.jurdana@vszi.upr.si damage to stem cells has serious repercussions because such cells are programmed to divide many times to maintain a large population and if they lose their reproductive integrity, they and their potential descendents are lost from the population. Thus, cells on the path to differentiation appear to be more radioresistant than stem cells. ${ }^{1}$

Radiation effects on tissue are divided into early and late effects, which show quite different patterns of response to fractionations and dose-response relations. Acute damage is rapidly repaired because of rapid proliferation of stem cells and may be completely reversible. ${ }^{2}$ Late effects appear after a delay of months or years and occur predominantly in slowly proliferating tissues, such as lung, kidney, heart, liver and central nervous system. Late damage may improve but it is never completely repaired and may result from a combination of vascular damage and loss of parenchymal cells. ${ }^{2}$ 


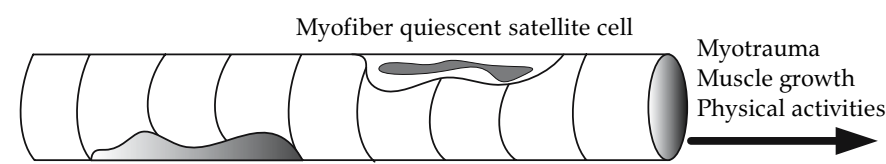

Myonuclei

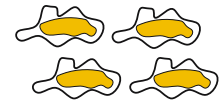

Satellite cell activation and proliferation.

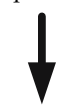

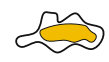

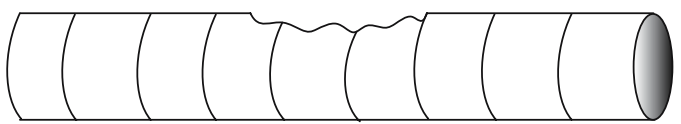

Chemotaxis to injured fiber

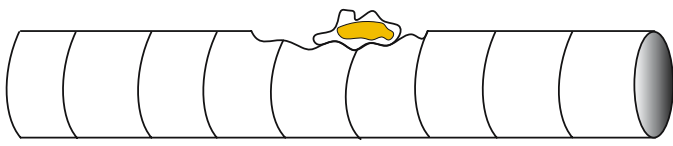

Fusion to damaged myofiber (hypertrophy)

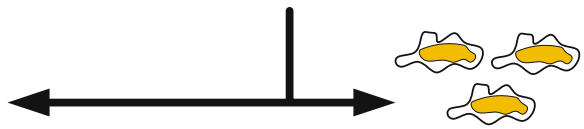

Fusion to produce new myofibres.

Figure 1. Satellite cell response to skeletal muscle trauma and muscle growth. Skeletal muscle trauma or injury may be minor (resistance exercise) or may be more extensive (muscular dystrophy). In response to both stimuli, satellite cells become activated and proliferate. Satellite cells will migrate to the damaged region and fuse to the extending myofiber or align and fuse to produce a new myofiber.

Casarett and Harris suggest a classification of mammalian cell radiosensitivity based on histopathologic observation. ${ }^{3}$ In terms of radiosensitivity, parenchymal cells fall into four categories, from the most sensitive to the most resistant. Adult muscle and nerve cells belong to the last, $4^{\text {th }}$ group, in which radiosensitivity is low and cells are highly differentiated.

It is generally assumed that adult nervous and skeletal muscle tissues are highly radioresistant. This may be true when based on the criterion of morphological damage that is manifested within a few months. The development of damage induces changes in muscle satellite cells, differentiated muscle cells and the neuromuscular junction.

\section{Effect of radiation on muscle satellite cells}

Satellite cells, a population of undifferentiated tissue-specific stem cells, were first identified in 1961 by Mauro. ${ }^{4}$ They are located at the periphery of the mature, multinucleated myotube and comprise only about $2 \%$ of the total nuclei of normal adult skeletal muscle $e^{4,5,6}$ and are normally nonproliferative.

Satellite cells have only one nucleus and can replicate by dividing. As the satellite cells multiply, some remain as organelles in the muscle fiber, whereas the majority differentiate and fuse to muscle fibers to form new muscle protein stands and/or repair damaged fibers (Figure 1). ${ }^{6}$ Muscle cell myofibrils will thus increase in thickness and number. After fusion with the muscle fiber, some satellite cells serve as a source of new nuclei to supplement the growing muscle fiber.

Satellite cells are constantly replenished during a lifetime, although there is a decline in satellite cell numbers and a reduced proliferative capacity in aged individuals. ${ }^{7,8}$ In response to stimuli such as myotrauma upon injury or muscle growth ${ }^{7}$, or when skel- 
etal muscle tissue is heavily used during physical activities ${ }^{9}$, satellite cells become activated and they turn into proliferating myoblasts. $^{7}$

Muscle induced myotrauma initiates an immune response, resulting in the influx of macrophages into the damaged region. After acute injury, macrophage infiltration peaks within 48 h. ${ }^{10}$ In the absence of a macrophage response, muscle regeneration is absent; in the presence of an enhanced macrophage response, there is an increase in satellite cell proliferation and differentiation. ${ }^{11}$

The purpose of the immune response is to contain, enclose the damage, repair the damage and clean up the injured area of waste products. The immune system causes a sequence of events in response to injury of the skeletal muscle. Macrophages, which are involved in phagocytosis of the damaged cells, move to the injury site and secrete cytokines, growth factors and other substances that regulate the satellite cell pool. ${ }^{12}$ Cytokines are responsible for cellto-cell communication and stimulate the arrival of lymphocytes, neutrophils, monocytes and other healer cells to the injury site to repair the injured tissue. ${ }^{13}$ The three important cytokines relevant to myotrauma are Interleukin-1 (IL-1), Interleukin 6- (IL-6) and tumor necrosis factor $\alpha$ (TNF- $\alpha$ ). They are responsible for protein breakdown, removal of damaged muscle cells, and increased production of prostaglandins.

Growth factors are highly specific proteins, which include hormones and cy- tokines that are involved in muscle hypertrophy ${ }^{14}$ and in the process of muscle regeneration: These processes result in regulation (activation) of the satellite cell population, which is controlled by growth factors and a sequence of intracellular events following binding of growth factors to their membrane receptors. ${ }^{15}$ This biological process often leads to an increase in muscle fiber cross-section area or hypertrophy. Increased muscle mass could restore muscle strength and prevent injuries. This is of particular importance in cachectic patients. Cachexia is a form of wasting that affects $50 \%$ of cancer patients. ${ }^{16}$ Increased muscle strength in cachectic patients may improve quality of life, improve response to cancer therapy and increase life span.

Radiation inhibits regeneration and muscle hypertrophy by damaging satellite cells. Radiation is thought to prevent satellite cell mitosis by causing breaks in strands of the cell's DNA. If a break occurs only on a single strand, the damage can be repaired by polymerases using the complementary strand as a template. If damage occurs at the same point on both strands, however, the deletion may be irreparable, which can lead to mitotic failure and cell death. ${ }^{17}$ Low levels of radiation, which should disable mitotically active satellite cells but not the post-mitotic myonuclei of adult skeletal muscle, prevent compensatory hypertrophy (Figure 2). Following irradiation, a sufficient number of satellite cells were apparently unavailable either to fuse to form new fibers during regeneration or to fuse to

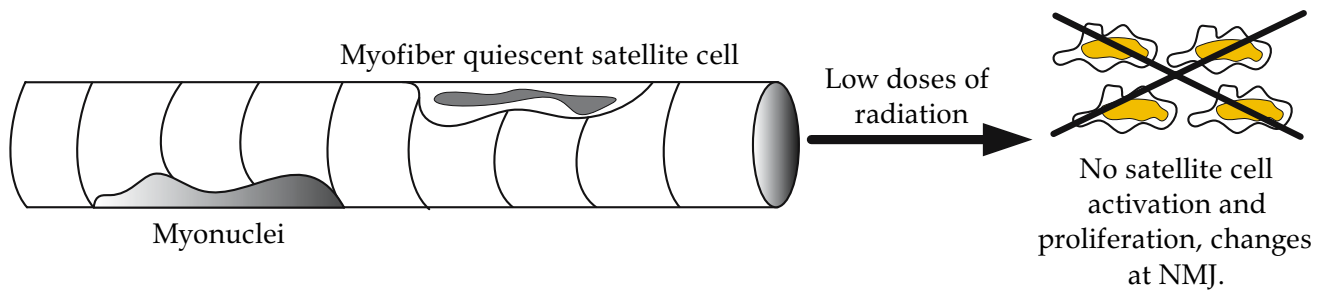

Figure 2. Satellite cells response to low doses of radiation. Low doses of radiation prevent muscle hypertrophy by impairing mitotically active satellite cells and induce changes in polysynaptic systems. 


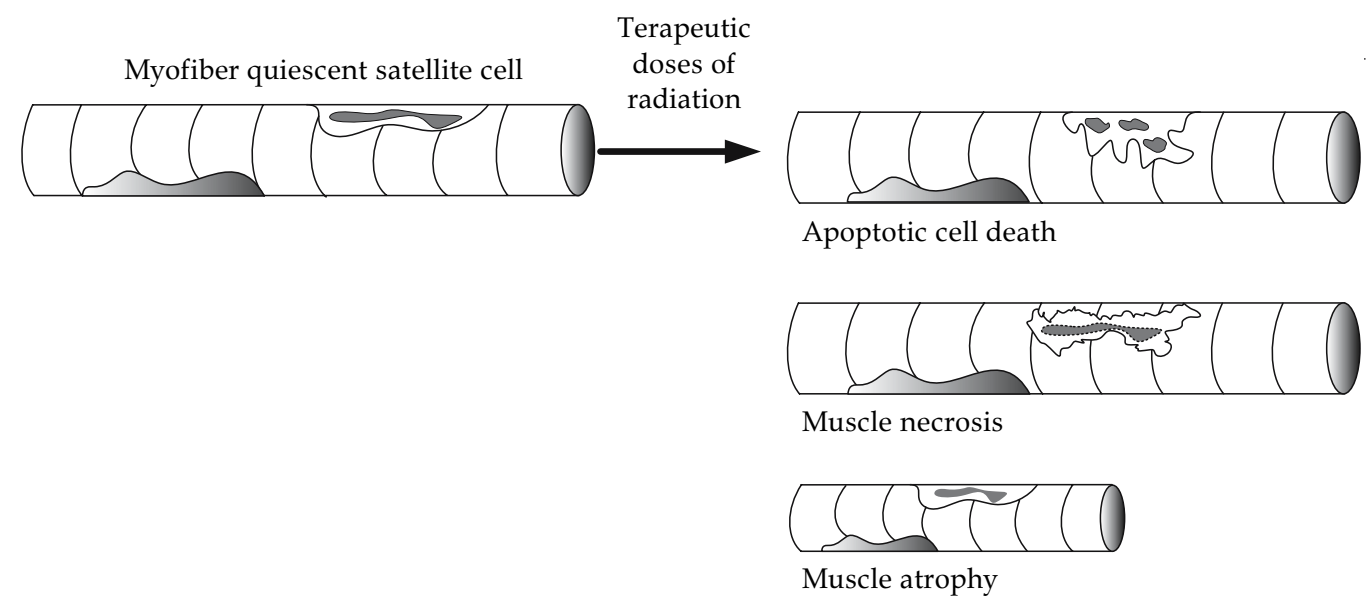

Figure 3. Skeletal muscle fiber response to therapeutic doses of radiation. Higher doses of radiation may induce apoptotic cell death and muscle necrosis, due to damage of blood capillaries and connective tissue. Muscle atrophy, resulting in myofiber degeneration and reduction of satellite cell population, was also observed.

overloaded fibers to allow hypertrophy occurring during overload. ${ }^{18}$

Olivé et al. showed that gamma irradiation (2 Gy) affects single skeletal muscle cells (satellite cells or myoblast) during development or cells in small clusters and induces its apoptosis. Since the effects of gamma rays are suppressed when cycloheximide is injected immediately after radiation, it can be suggested that gamma ray induced cell death is an active process associated with protein synthesis (extremely condensed chromatin). ${ }^{19}$

Transforming growth factor $-\beta$ (TGF- $\beta$ ) is the prototypical family member of cytokines. The function of members of the TGF- $\beta$ superfamily is to inhibit muscle proliferation and differentiation. ${ }^{20}$ Following irradiation, the induction of a strong inflammatory response, fibrosis and vascular changes that associate with late radiation effects are linked to the action of TGF- $\beta$. Myostatin (GDF-8), a member of the (TGF$\beta)$ superfamily is a specific negative regulator of skeletal muscle mass. ${ }^{21}$ It has been demonstrated that myostatin inhibitors could provide therapeutic benefit in diseases for which muscle mass is limiting. ${ }^{22}$
Many other factors may be involved in the regulation of satellite cells in skeletal muscle. Hematopoietic cells are responsible for constant maintenance and immune protection of every cell type of the body, including skeletal muscle. Radiation induces IL-1 and IL-6, which act as a radioprotectors of muscle and hematopoietic cells. TGF- $\beta$ may down-regulate IL- 1 and TNF- $\alpha$ and increase damage to hematopoietic tissue. The expression of TNF- $\alpha$ following radiation is believed to be regulated at the transcriptional level and involves the protein kinase C-dependent pathway.

\section{Effects of radiation on differentiated muscle cells}

Human striated muscle is highly radio-resistant on the criterion of morphological changes; however, delayed necrosis of muscle is observed after therapeutic doses of radiation (Figure 3). ${ }^{23}$ The delayed necrosis of muscle may be primarily due to damage to blood capillaries and connective tissue. A cumulative dose of 4500 rads (45 Gy) produced detectable damage in mus- 
cle 3 years post-radiation in patients with otherwise good general health. However, in patients suffering from carcinomatous cachexia or other debilitating diseases, muscle necrosis was constantly observed following exposure to about 2000 rads (20 Gy) after a similar latent period. ${ }^{24}$ Lefaix et al. demonstrate that muscle fibrosis is a common and irreversible late effect of radiation on skeletal muscle. ${ }^{25}$ A thyrotoxicosis condition (hypermetabolic clinical syndrome resulting from serum elevation in thyroid hormone T3 and T4 levels), produces muscle necrosis within 5 months after 2200 rads (22 Gy), whereas the control subjects, irradiated with same dose, did not show any muscle lesion over a period of 2 years. Children who have been treated with radiotherapy for a malignant disease may show muscular atrophy after a long latent period. This is seen in the back muscles of long-term survivors of Wilm's tumor and in the neck muscles of survivors of Hodgkin's disease, after radiation therapy. ${ }^{23}$

\section{Neuromuscular junction}

A neuromuscular junction (NMJ) is the synapse or junction of the axon terminal of a motoneuron with the motor end plate, the highly-excitable region of muscle fiber. The plasma membrane responsible for initiation of action potentials across the muscle's surface ultimately causes muscle contraction. In vertebrates, the signal passes through the neuromuscular junction via the neurotransmitter acetylcholine (Ach).

The precise mechanism of the radiation effects on NMJ is unknown. It has been suggested that temporary changes in membrane permeability for potassium and sodium, the liberation of pharmacologically active substances such as serotonin and ACh, and interaction between lipoproteins and free radicals, may contribute to synaptic changes after radiation. ${ }^{26}$ Changes in membrane permeability for sodium and potassium influence the activity of electrogenic $\mathrm{Na}^{+} / \mathrm{K}^{+}$pump. The pump in the skeletal muscle membrane generates a small electric current by extruding three $\mathrm{Na}^{+}$ions, while taking in only two $\mathrm{K}^{+}$ions in each cycle, and is responsible for action potential propagation. ${ }^{27}$ Modulation of its activity leads to alteration of muscle excitability and contractility.

In general, low doses of radiation result in dose-rate dependent stimulation of synthesis of ACh, which is released by branching motor nerves. This causes Ach receptorinduced postsynaptic potentials and positively regulates the localization and stabilization of developing synaptic contacts and cellular uptake of 5- hydroxytryptamine (serotonin). Neurophysiological studies with intracellular electrodes have shown that irradiation with above 500 rads (5 Gy) reduces metabolic activity, ${ }^{28}$ irradiation of 600 rads (6 Gy) produces significant changes in monosynaptic excitory and postsynaptic potentials. ${ }^{29}$ Doses of 200 rads (2 Gy) of whole body irradiation cause changes in polysynaptic systems. ${ }^{30}$

\section{Radiation and aging sarcopenia}

It is now well established that cancer and aging are connected and that they share the same molecular events. In addition, it has been shown that radiation therapy accelerates the aging process in animals, but this remains controversial in humans. ${ }^{31,32,33}$

In humans, aging is a complex process that determines many physical and metabolic alterations correlated to the accumulation of oxidative damages in different tissues. Sarcopenia is an age-related nonpathological condition that includes a progressive loss of mass and strength in skeletal muscle, associated with a decline in the 
fibers' functional capability. ${ }^{34}$ A decrease in satellite cell number and/or proliferative capacity has been used to explain this phenomenon. Aging negatively affects the immune response, resulting in a decrease in inflammatory factors and macrophages. ${ }^{35}$ $(\mathrm{Ca})^{2+}$ homeostasis also seems to be modified. ${ }^{36}$

The detrimental effects of aging on muscle have been shown to be restrained or even reversed with regular resistance exercise. Resistance exercise also improves the connective tissue harness surrounding muscle, thus being most beneficial for injury prevention and in physical rehabilitation therapy. ${ }^{37}$

The free radical theory of aging suggests a crucial role for free radicals produced by external factors, as well as radiation. The mechanism that is responsible for free radical - mediated damage in an organism is superoxide overproduction by mitochondria, which causes the inhibition of nitric oxide formation and bioavailability, one of the principal characteristics of aging. ${ }^{38}$

Cancer and aging share many common metabolic pathways and mediators during muscle wasting. Both cancer cachexia and aging sarcopenia may represent targets for future promising clinical investigations. ${ }^{39}$

\section{Conclusion}

Radiation, which should disable mitotically active satellite cells, prevents compensatory hypertrophy and nearly abolishes small fiber formation in the overloaded mammalian skeletal muscle. Irradiation may prevent hypertrophy by impairing activation, proliferation and/or differentiation of satellite cells. This suggests that satellite cell viability is essential for muscle hypertrophy. ${ }^{40}$

Therapeutic doses of radiation induce muscle atrophy in children and irreversible fibrosis in adult skeletal muscle. Little is known and little work has been done on the precise mechanism of how skeletal muscle adapts after radiation.

The mechanism underlying changes in the neuromuscular junction has not yet been completely clarified, and therefore calls for further investigation, especially of $\mathrm{Na}^{+} / \mathrm{K}^{+}$pump expression and its activity.

More research should be devoted to the understanding of muscle wasting mediators, both in cancer and aging. Identification of common mediators in particular may prove to be a good therapeutic strategy for prevention and treatment of wasting in cancer cachexia and during normal aging.

\section{References}

1. Hall, EJ, Giaccia AJ. Radiobiology for the radiologist. Clinical response of normal tissues. Philadelphia: Lippincott Williams \& Wilkins; 2006. p: 339.

2. Hall, EJ, Giaccia AJ. Radiobiology for the radiologist. Clinical response of normal tissues. Philadelphia: Lippincott Williams \& Wilkins; 2006. p: 340.

3. Casarett G, Haris RJC. Cellular basis and aetiology of late somatic effects of ionizing radiation. In Casaret H, Hariss RJC, editor. Concept and criteria of radiologic ageing. New York: Academic Press; 1963. p. 496-506.

4. Mauro A. Satellite cell of skeletal muscle fibers. J Biophys Biochem Cytol 1961; 9: 493-8.

5. Collins CA, Olsen I, Zammit PS, Heslop I, Petrie A, Partridge TA, Morgan JE. Steam cell function, self-renewal and behavioral heterogeneity of cells from the adult muscle satellite cell niche. Cell 2005; 122: 289-301.

6. Charge SB, Rudnicki MA. Cellular and molecular regulation of muscle regeneration. Physiol Rev 2004; 84: 209-38.

7. Bischoff R. The satellite cell and muscle regeneration. In: Engel AG, Frazini-Armstrong C, editor. Myology. New York: McGraw-Hill; 1994. p. 97-118.

8. Seale P, Rudnicki MA. A new look at the origin, function, and "stem-cell" status of muscle satellite cells. Dev Biol 2000; 218: 115-24. 
9. Appell HJ, Forsberg S, Hollman W. Satellite cell activation in human skeletal muscle after training: evidence for muscle fiber neoformation. Int $J$ Sports Med 1989; 4: 297-9.

10. Vierck J, O’Reilly B, Hossner K, Antonio J, Byrne $\mathrm{K}$, Bucci L, Dodson M. Satellite cell regulation following myotrauma caused by resistance exercise. Cell Biol Int 2000; 24: 263-72.

11. Lescaudron L, Peltekian E, Fontaine-Perus J, Paulin D, Zampieri M, Garcia L, et al. Satellite cells as the source of nuclei in muscles of growing rats. Anat Rec 1971; 170: 421-35.

12. Nathan CF. Secretory products of macrophages. J Clin Invest 1987; 79: 319-26.

13. Pedersen BK. Exercise immunology. New York: Chapman \& Hall; 1997.

14. Adams GR, Haddad F. The relationships among IGF-1, DNA content, and protein accumulation during skeletal muscle hypertrophy. J Appl Physiol 1996; 81: 2509-16.

15. Grounds MD. Muscle regeneration: molecular aspects and therapeutic implications. Curr Opin Neurol 1999; 12: 535-43.

16. Tisdale MJ. Clinical trials for the treatment of secondary wasting and cachexia. Nutrit 1999; 129: S243-6.

17. Denekamp J, Rojas A. Cell kinetics and radiation pathology. Experientia 1989; 45: 33-41.

18. Rosenblatt JD, Yong D, Perry DJ. Satellite cell activity is required for hypertrophy of overloaded adult rat skeletal muscle. Muscle Nerve 1994; 17: 608-13.

19. Olivé M, Blanco R, Rivera R, Cinos C, Ferrer I. Cell death induced by gamma irradiation of developing skeletal muscle. J Anat 1995; 187(Pt 1): 127-32.

20. Allen RE, Boxhorn LK. Regulation of skeletal muscle satellite cell proliferation and differentiation by transforming growth factor -beta, insulin-like growth factor I, and fibroblast growth factor. J Cell Physiol 1989; 138: 311-5.

21. McPherron AC, Lawler MA, Lee SJ. Regulation of skeletal muscle mass in mice by a new (TGF- $\beta$ ) superfamily member. Nature 1997; 387: 83-90.

22. Whittemore LA, Song K, Li X, Aghajanian J, Davies M, Girgenrath S, Hill JJ, et al. Inhibition of myostatin in adult mice increases skeletal muscle mass and strength. Biochem Biophys Res Commun 2003; 300: 965-71.
23. Zeman W, Soloma M. Effects of radiation on striated muscle. In: Berdijs CC, editor. Pathology of irradiation. Baltimore: Williams \& Wilkins; 1971. p. 185.

24. Prasad KN. Handbook of radiobiology. Radiation damage of other organ systems. Boca Raton: CRC press; 1995. p: 181-92.

25. Lefaix JL, Delanian S, Leplat JJ, Tricaud Y, Martin M, Hoffschir D, et al. Radiation induced cutaneomuscular fibrosis: major therapeutic efficacy of liposomal $\mathrm{Cu} / \mathrm{Zn}$ superoxide dismutase. Bull Cancer, 1993; 80: 799-807.

26. Gangloff $\mathrm{H}$, Hug $\mathrm{O}$. The effects of ionizing radiation on the nervous system. Adv Biol Med Phys; 1965: 10: 1 .

27. Post RL, Jolly DC. The linkage of sodium, potassium, and ammonium active transport across the human erythrocyte membrane. Biochim Biophys Acta 1957; 25: 118-28.

28. Zeman W, Ordy JM, Samorajski T. Modification of acute irradiation effect of cerebellar neurons of mice by actinomycin D. Exp Neurol 1968; 21: 57.

29. Sato M, Augustin G, Stahl W. The effects of ionizing radiation on spinal cord neurons. In: Haley $\mathrm{TJ}$, Snider RS, editor. Response of the nervous system to ionizing radiation. New York: Academic Press; 1962. p.: 57.

30. Gangloff H. Hippocampal spike activity following low doses of radiation. In: Haley JF, Snider RS, editor. Response of the nervous system to ionizing radiation. Boston: Little Brown; 1964. p. 574.

31. Johnson HA. Age and senistivity to radiation life shortening. Radiat Res 1964; 23: 19.

32. Failla G, McClement P. The shortening of life by chronic whole-body irradiation. Am J Roentgenol Radium Tehr Nucl Med 1957; 78: 964.

33. Hursh JB, Noonon TR, Casarett GW, Vanslyke F. Reduction of life span of rats by roentgen irradiation. Am J Roentgenol Radium Tehr Nucl Med 1955; 74: 130.

34. Mishra SK, Misra V. Muscle sarcopenia: an overview. Acta Myol 2003; 22: 43-7.

35. Danon D, Kowatch MA, Roth GS. Promotion of wound repair in old mice by local injection of macrophages. Proc Natl Acad Sci USA 1989; 86: 2018-20. 
36. Fulle S, Di Donna S, Puglielli C, Pietrangelo T, Beccafico S, Bellomo R, et al. Age-dependent imbalance of the antioxidative system in human satellite cells. Exp Geront 2005; 40: 189-97.

37. Clarke MS. The effects of exercise on skeletal muscle in the aged. J Musculoskelet Neuronal Interact 2004; 4:175-8.

38. Afanasev IB. Free radical mechanisms of ageing processes under physiological conditions. Biogerontol 2005; 6: 283-90.

39. Argilés JM, Busquets S, López-Soriano FJ, Figueras M. Physiology of sarcopenia. Similarities and differences with neoplasic cachexia (muscle impairments in cancer and ageing. Nutr Hosp 2006; 21: 38-45.

40. Phelan NJ, Gonyea WJ. Effect of radiation on satellite cell activity and protein expression in overloaded mammalian skeletal muscle. The Anat Rec 1997; 247: 179-88. 